Case Report

\title{
Clinical management of a rare Peters' anomaly-induced secondary childhood glaucoma: A case report
}

\author{
Eva Imelda ${ }^{1,2^{*}}$ and Fany Gunawan ${ }^{3}$ \\ ${ }^{1}$ Department of Ophthalmology, Faculty of Medicine, Universitas Syiah Kuala, Banda Aceh, Indonesia; ${ }^{2}$ Dr Zainoel \\ Abidin Hospital, Banda Aceh, Indonesia; ${ }^{3}$ Cicendo Eye Hospital, Bandung, Indonesia
}

*Corresponding authors: evaimeldaspmpo@gmail.com

\begin{abstract}
Childhood glaucoma is a rare disorder that occurs from birth until teenage years caused by an abnormality of aqueous humor pathways. About $50-70 \%$ of Peters' anomaly is accompanied by secondary childhood glaucoma. The presence of glaucoma will affect the prognosis. We reported the evaluation and treatment of secondary childhood glaucoma due to Peters' anomaly. A 5 months-old boy was presented with the complaint of a enlarged left eye since 3 months old. The complaint was accompanied by a watering eye and frequently closed upon light exposure. The left eye looked opaquer than contralateral. Examination under anesthesia showed that the intraocular pressure (IOP) was $35 \mathrm{mmHg}$ in the left eye and the corneal diameter was $14 \mathrm{~mm}$. Other findings were keratopathy, diffuse corneal edema, buphthalmos, shallow anterior chamber, anterior synechiae, and linear slit shaped pupils in the nasal region. Patient was treated with ophthalmic timolol maleate which was later followed by trabeculectomy. After 1 week post-surgery, IOP assessment by palpation suggested the right eye within normal range while the IOP of left eye was higger than normal. Blepharospasm, epiphora, photophobia, bleb on superior, subconjunctiva bleeding, buphthalmos, keratopathy, minimal corneal edema, anterior chamber with shallow image, and posterior synechia were found in left eye anterior segment. In conclusion, trabeculotomy and trabeculectomy are recommended if there is no reduction of IOP observed after receiving timolol maleate therapy. The choice of surgical management is dependent on the feasibility of the protocol.
\end{abstract}

Keywords: Pediatric glaucoma, secondary childhood glaucoma, Peters' anomaly, anterior segment dysgenesis, corneal opacity

\section{Introduction}

$P_{\text {eters' anomaly is a congenital ocular anomaly with manifestations covering corneal opacity }}$ and anterior segment dysgenesis which emerge during ocular development. Most cases of Peters' anomaly take place sporadically, except in several cases where hereditary patterns were found as recessive and dominant autosomal gene $[1,2]$. Peters' anomaly is cause by genetic mutation having a role in the development of eye anterior segment, including PAX6 (11p13), PITX2 (4q25-26), CYP1B1 (2p22-21), and FOXC1 (6p25) [1, 3]. The incidence of Peters' anomaly at birth is extremely rare, diagnosed in 1 out of 70,00o live births in the United States [4].

Characteristics of Peters' anomaly are the presence of central corneal opacity with defect in the posterior stroma, Descemet membrane, and corneal endothelium. The peripheral corneal 
region tends to be clearer. Other clinical characteristics include the presence of iris attachment stretching from collaret to posterior surface with corneal opacity [3, 5]. Corectopia is often found along with a shallow anterior chamber. The size and degree of corneal opacity vary from lines up to covering the entire corneal part which could lead to deprivation amblyopia. More than $60 \%$ of Peters' anomaly cases take place bilaterally [6].

Management of Peters' anomaly is based on the degree of severity and visual prognosis [3, $7,8]$. The management covers medicamentosa therapy and surgery. Medicamentosa therapy includes drugs to control the eye pressure, while surgical therapy covers penetrating keratoplasty and peripheral iridectomy. Penetrating keratoplasty is recommended for patients with bilateral corneal opacity or thick opacity aiming to improve the visual axis transparency [9]. The surgery is better to be performed within the ages of 2 to 12 months old to prevent amblyopia. Penetrating keratoplasty in pediatric patients is still a challenging procedure because it requires difficult techniques and has the risks of side effects from narcosis, rejection, infection, and implant failure [7, 8]. Furthermore, the procedure needs complex postoperative management. The success rate of keratoplasty in Peters' anomaly is $87.5 \%$ in type I and $14.2 \%$ in type II [8]. Penetrating keratoplasty could not be done if the corneal neovascularization is present, uncontrolled glaucoma, infection, or a poor layer of tear film. Peripheral iridectomy could be done in cases with mild corneal opacity without the company of lens abnormalities [10].

Ocular abnormalities could be developed after the occurrence of Peters' anomaly, including glaucoma that is found in 50-70\% cases [11]. Childhood glaucoma is a heterogenous disease group caused by impaired aqueous humor outflows, either isolated or correlated with ocular or other systemic diseases. The World Glaucoma Association classifies the disease group into primary and secondary childhood glaucoma $[12,13]$. Primary congenital glaucoma (PCG) is the most common type of childhood glaucoma. As many as 2 in 3 cases occurred bilaterally with male predominance reaching $65 \%$ of the total cases. PCG onset often occurs during the first year since birth (80\% cases), where only $25 \%$ cases were diagnosed since birth [14]. Clinical manifestation of PCG include epiphora, blepharospasm, and photophobia (classic triad of PCG). The manifestations were followed by corneal opacity, enlargement, with Haab striae (curvilinear lines on the Descemet membrane), iris insertion more into anterior, and peripheral iris stroma hypoplasia. The entire eye could enlarge, contributing to the its appearance resembling buffalo's eyes (buphthalmos) and pseudoproptosis [14].

Peters' anomaly accompanied with glaucoma could be managed through filtration surgery, angle surgery, cryoablation, and a tube shunt to reduce the eye pressure and to improve corneal transparency $[8,10,11]$. The size and severity of cornea opacity vary from lines to the entire part of the cornea, capable of causing deprivation amblyopia. The involvement of glaucoma could affect patients' prognosis. Moreover, the prognosis is also affected by the type of operative technique and duration $[6,11]$. Herein, we reported the management a patient with rare secondary childhood glaucoma due to Peters' anomaly. The use of surgical management in Peters' anomaly-assocaited glaucoma still do not show a high success rate [8]. Only a retrospective study from the United States reported the management for pediatric patients with glaucoma and Peters' anomaly [7]. Therefore, there is an urgency of reporting the management of glaucoma in Peters' anomaly.

\section{Case}

A 5 month-old boy was brought to the Pediatric Ophthalmology Clinic of Cicendo Mata Hospital on May 16, 2019 with a complaint of an enlarged left eye which had been present since 3 months old. The mother conveyed that the complaint was followed by a watery condition, and the patient often closed his eyes in presence of bright light. The left eye appeared opaquer than the other eye. The patient had never been brought to or received treatment from ophthalmologist. Histories of any systemic or ocular diseases were not present. The father stated the patient's left eye had appeared smaller than the other eye since birth. The patient was the first child with normal pregnancy and delivery with the absence of delivery trauma. After delivery, the child cried immediately and had a body weight of 2.7 kilograms. The mother had periodical check-ups during the pregnancy ans did not report a history of fever or other diseases nor consumption of 
alcohol or drugs during pregnancy. The patient's growth was normal and was in accordance with his age. The patient had complete immunization. Glaucoma or other similar complaints were not found in the histories of patient's family tree.

Physical observations of the patient revealed that the body weight $(6.2 \mathrm{~kg})$ and length $(60.5$ $\mathrm{cm}$ ) were still within the normal range. Ophthalmological examination suggested that the patient's eyes were able to fix and follow an object. Images of the patient's eyes recorded in 1, 3, and 5 months old are presented in Figure 1A-C. Further assessment on intraocular pressure (IOP) by palpation resulted the IOP assessment by palpation suggested the right eye within normal range while the IOP of left eye was higger than normal. The right eye anterior segment was observed in a normal range with a corneal diameter of $11 \mathrm{~mm}$. Meanwhile, blepharospasm, epiphora, photophobia, buphthalmos, diffuse corneal edema with Haab striae and 13-mm long corneal diameter were found in the left eye anterior segment. Visualizations of the anterior chamber depth, pupil, iris, and lens were difficult to assess owing to the corneal edema. Examination on the posterior segment by indirect fundoscopy on the right eye suggest the transparent media, rounded papillae with firm borders, cup-to-disc ratio of 0.3 , arteriole-tovenule width ratio of 2:3, flat retina, and positive fundus reflex; while opaque media was observed on the left eye.

Ultrasonography (USG) examination on the right eye showed an image in a normal rage with an axial length of $16.55 \mathrm{~mm}$ (Figure 1D). Meanwhile, the left eye was observed to have an echogenic vitreous cavity, obscured shape, low to medium reflectivity, medium to high mobility, intact retina, choroid, and sclera, and axial length of $19.55 \mathrm{~mm}$. USG of the left eye (Figure 1E) exhibited a macrophthalmia image with mild vitreous opacity associated with vitreous fibrosis or inflammation cells.
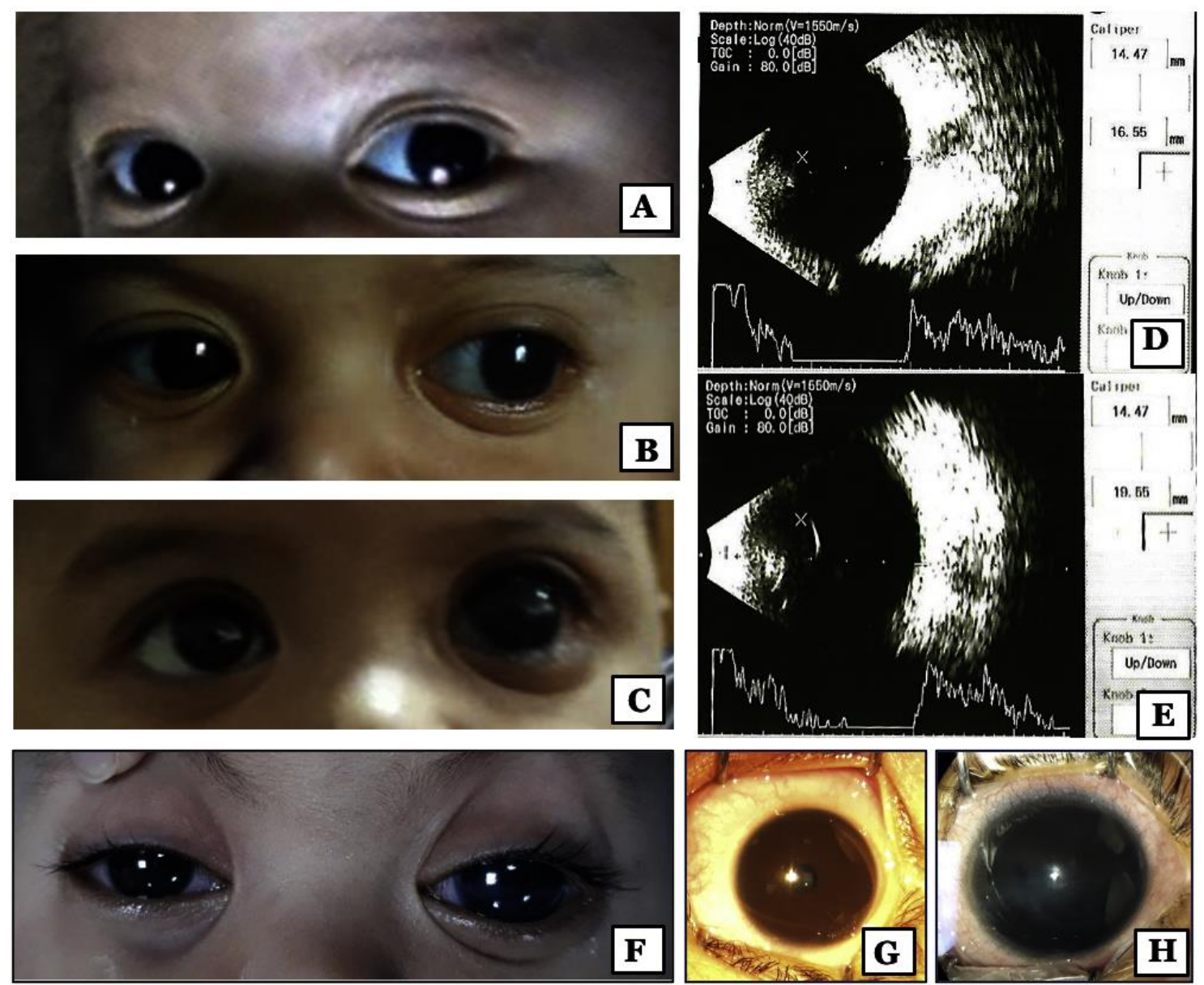

Figure 1. Clinical images of patient's eyes at different ages before the treatment and ultrasonography of the eyes. Clinical images of patient eyes aged 1 month (A), 3 months (B), and 5 months (C). The ultrasonography images of the patient's right eye (D) and left eye (E). Preoperative clinical image of the patient on June 24, 2019 (F); right eye (G) and left eye (H) anterior segment. 
The patient was diagnosed with PCG and the condition was managed by the administration of ophthalmic timolol maleate $0.25 \% \mathrm{w} / \mathrm{v}$. The examination under anesthesia (EUA) of both eyes under inhalation along with a consideration of combination of trabeculotomy and trabeculectomy for the left eye under general anesthesia were planned. The patient was referred to the Department of Pediatric Health for preoperative assessment, where systemic disorders were not found.

EUA of both eyes was performed on June 24, 2019 revealing the right eye IOP of $15 \mathrm{mmHg}$, corneal diameter of $11 \mathrm{~mm}$, and the anterior and posterior segments were within the normal range. The left eye IOP was $35 \mathrm{mmHg}$, corneal diameter of $14 \mathrm{~mm}$, calm palpebra, ciliary injection, keratopathy, corneal edema diffuse, and buphthalmos, while the anterior chamber, pupil, iris, and lens were difficult to assess. The patient was then prepared for a combination procedure of trabeculotomy and trabeculectomy on the left eye under narcosis. Preoperative clinical images of the patient eyes are presented in Figure 1F-H. During the operation, it was retrieved with minimal corneal edema to unveil the shallow anterior chamber, anterior synechia, as well as a linear slit-shaped pupil on the nasal region. The patient was diagnosed with segment dysgenesis of left eye anterior (Peters' anomaly) as well as left eye secondary childhood glaucoma and the patient was decided for trabeculectomy, where the steps are depicted in Figure 2.
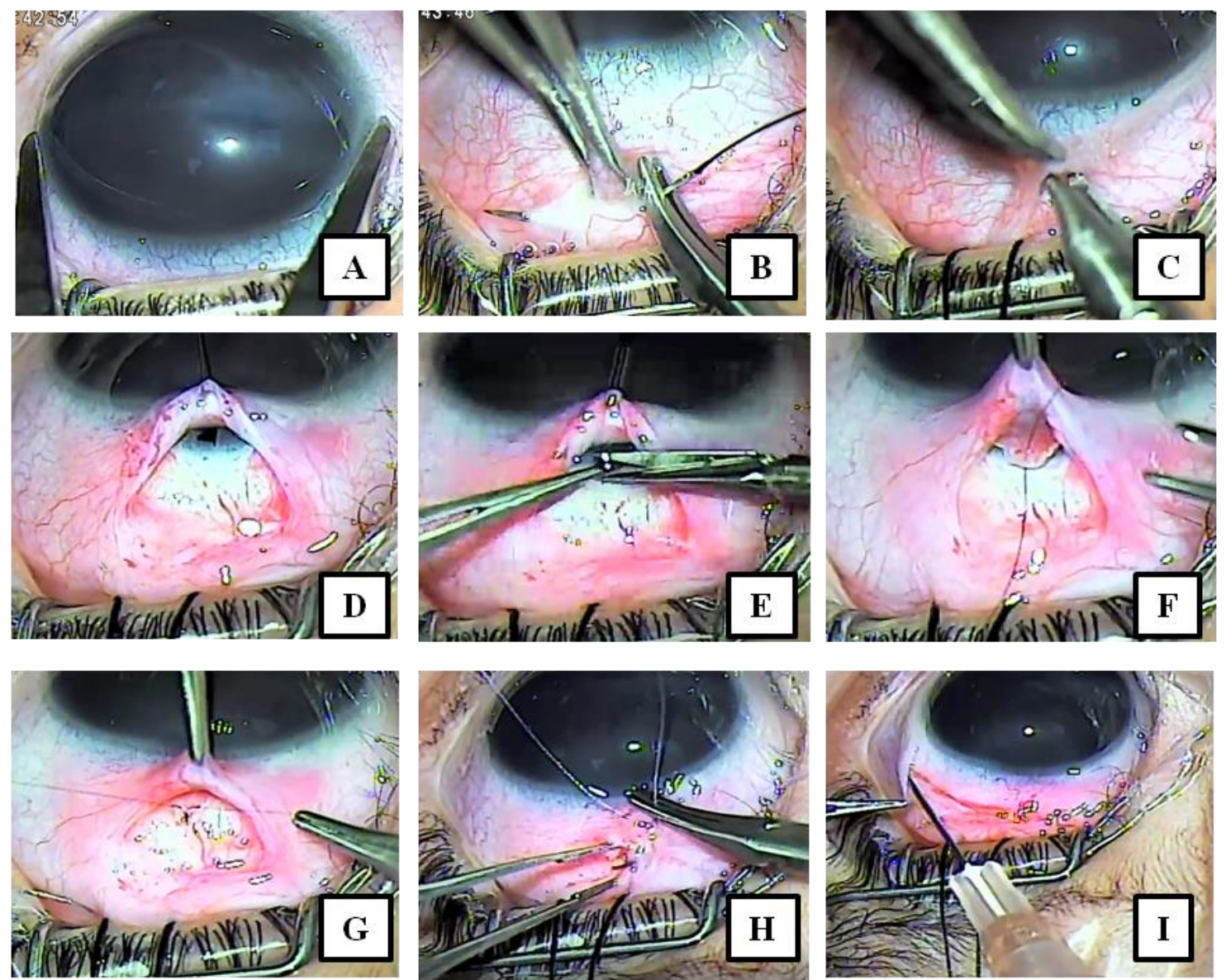

Figure 2. Steps of trabeculectomy performed on the patient: (A) Corneal diameter measurement (14 mm); (B) Installation of rectus control; (C) Peritomy with limbus basis; (D) Preparation sclera flap and sclerotomy; (E) Iridectomy; (F, G) Flap sclera sewing; (H) Conjunctiva sewing, and (I) Subconjunctival injection of gentamicin and dexamethasone

Postoperative therapies included cefadroxil syrup $2 \times 125 \mathrm{~mL}$ peroral, paracetamol syrup 3 x $80 \mathrm{mg}$ peroral, ophthalmic levofloxacin $6 \times 1$ drops, ophthalmic prednisolone acetate $8 \times 1$ drop, and ophthalmic homatropine $2 \% 2 \times 1$ drop all for left eye. The patient was scheduled for follow-up visit 7 days postoperative.

The patient was presented for postoperative control to the Pediatric Ophthalmology Clinic on July 1, 2019 with vision of oculus for both eyes were fix and followed by an object 
examination. OIP assessment by palpation showed the right eye within normal range while the IOP of left eye was higger than normal. The anterior segment of the left eye was observed with blepharospasm, epiphora, photophobia, bleb on superior, subconjunctiva bleeding, buphthalmos, keratopathy, minimal corneal edema, anterior chamber with shallow image, and posterior synechia. A detailed visualization of the pupil, iris, and lens were still hard to assess. The patient was treated with ophthalmic timolol maleate $0.25 \% 2 \times 1$ drops, ophthalmic levofloxacin $6 \times 1$ drops, ophthalmic prednisolone acetate tapering off $6 \times 1$ drops for 1 week and $5 \times 1$ drops for the next one week, and ophthalmic homatropine $2 \% 2 \times 1$ drop. The patient was recommended for follow-up visit within the following two weeks. Prognosis for the patient were quo ad vitam dubia ad malam, quo ad sanationam dubia ad malam, and quo ad functionam dubia ad malam.

\section{Discussion}

Primary childhood glaucoma occurs due to the primary congenital abnormality from the angle of the anterior chamber. Based on its onset at the patient's age, primary childhood glaucoma could be differentiated into PCG and juvenile open-angle glaucoma [13]. Congenital ocular abnormalities correlated with glaucoma include: anterior segment abnormalities (such as but not limited to aniridia, Axenfeld-Rieger syndrome, Peters' anomaly, sclerocornea, microcornea, and congenital iris ectropion) as well as posterior segment abnormalities (such as persistent fetal vasculature (PFV), retinopathy of prematurity (ROP), familial exudative vitreoretinopathy (FEVR), and iris, retina, or ciliary tumor $[12,14]$.

Impairment of aqueous humor outflows might cause the increment of aqueous humor resistance allowing the IOP to elevate. The increase on IOP leads to corneal edema and corneal diameter enlargement stemming from gradual stretching. The patient reported in this case report was diagnosed with the occurrence of OS anterior segment dysgenesis stem from Peters' anomaly followed by OS secondary childhood glaucoma.

The development of the cornea depends on the neural crest migration emerging in 3 phases of anterior segment formation during week seven of the gestation period (Figure 3). The first phase is the formation of the corneal endothelium through the migration of the neural crest between surface ectoderm and lens. The second phase covers the migration of the peripheral neural crest between the newly formed corneal endothelium and surface ectoderm to form keratocyte which later forms corneal stroma. The last phase includes the formation of the iris stroma. Disruption on migration and separation of neural crest will cause the dysgenesis of anterior segment [15].
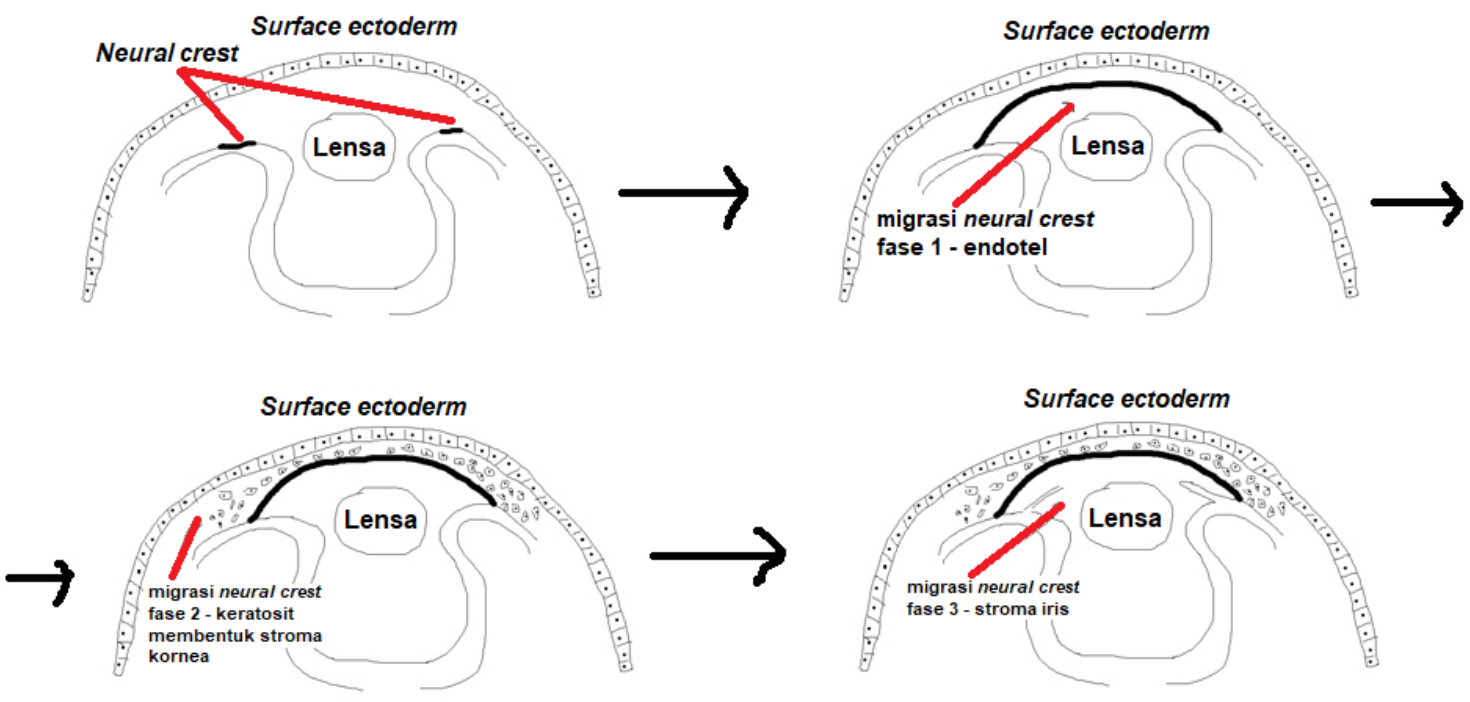

Figure 3. Formation phase of eye anterior segment. Reproduced under the terms of under the Creative Commons Attribution License 4.0, citing Chun et al. [16]. 
Clinical diagnosis is acquired based on characteristic findings during the observation of the ocular anterior segment. Additional examinations could be conducted, including ultrasonography B-scan, ultrasonography biomicroscopy, and genetic investigation. For the most part, Peters' anomaly is correlated with other ocular abnormalities such as glaucoma, cataracts, microphthalmia, sclerocornea, iris hypoplasia, aniridia, and coloboma. Of these, glaucoma is the most commonly found in Peter's anomaly patients [11]. It is ascribed to the fact that the shallow anterior chamber was derived from the dysgenesis structure of the anterior angle leading to the disruption of aqueous humor outflows. Herein, the patient had a clinical manifestation of glaucoma in the forms of corneal edema, buphthalmos, as well as IOP elevation. The examination of the anterior segment revealed the mild unilateral corneal opacity with a shallow anterior chamber, anterior synechia, and corectopia with linear slit on the pupil around nasal region.

Peters' anomaly can be distinguished into type I and II. Peters' anomaly type I is a less severe type with limited abnormalities on the iris, corneal endothelium, and Descemet membrane which are iridocorneal adhesion. Peters' anomaly type I often occurs in a unilateral manner with clear lens and better prognosis [3, 5]. Peters' anomaly type II, on the other hand, takes place bilaterally with thicker corneal opacity and is accompanied with the presence of lens abnormalities, keratolenticular adhesion, and cataracts [3, 6, 17]. Hence, the patient was diagnosed with Peters' anomaly type I with secondary childhood glaucoma on left eye. USG examination on the left eye was performed to obtain the posterior segment images along with eye length (which was $19.55 \mathrm{~mm}$ ), where the results suggest macrophtalmia with mild vitreous opacity stemming from vitreous fibrosis or inflammation cells $[5,6]$.

Differential diagnosis requiring consideration in the case of corneal opacities includes sclerocornea, ruptured Descemet membrane (due to forceps trauma or glaucoma congenital), ulcers, metabolic diseases (such as mucopolysaccharidosis), defected posterior cornea (such as Peters' anomaly and keratoconus posterior), corneal edema caused by glaucoma or congenital corneal dystrophy (such as congenital hereditary stromal dystrophy and congenital hereditary endothelial dystrophy), as well as dermoid. Sclerocornea and Peters' anomaly are dysgenesis of the anterior segment and share several common clinical characteristics; except in Peters' anomaly, peripheral corneal region appears clearer. Moreover, the cornea in sclerocornea is entirely opaque and flat [18]. That characteristic was not found in the patient of this study. Histories of delivery trauma, red eye, and rheum, or family histories with corneal opacity were not found in the patient. The anterior segment examination revealed a shallow anterior chamber with anterior synechia and corectopia. Ruptures in Descemet membrane, ulcers, metabolic diseases, primary congenital glaucoma, and congenital corneal dystrophy had normal anterior chamber depth leading to exclusion of intended diagnosis in this case.

Further differential diagnosis was conducted for other anterior segment dysgeneses such as Axenfeld-Rieger syndrome. This syndrome is characterized by the presence of posterior embryotoxon which is a shifting of the Schwalbe line position more into anterior from the limbus (Axenfeld anomaly) accompanied with iris abnormalities, such as iris hypoplasia, corectopia, polycoria, and ectropion uvea (Rieger anomaly). Its distinction with Peters' anomaly is cornea that is relatively clearer and the opacity is found in central cornea [19].

Systemic examination is important to identify the presence of syndrome or accompanying systemic disorders. As many as 60\% of Peters' anomaly cases are correlated with systemic disorders [3]. The abnormalities include short and less proportional postures, inhibited growth, facial dysmorphia and extremities, cardiovascular malformation, genitourinary disorders, and central nerve arrangements (Peters' plus syndrome) [3, 17]. Genetic consultation and comprehensive examination by pediatric departments are highly recommended. The patient in this case report had generalist status within the normal range, where the growth and development were in accordance with the age. The patient was also referred to the pediatric department, and no systemic disorders were found.

The patient still had IOP in the left eye as high as $35 \mathrm{mmHg}$ despite after receiving timolol maleate therapy $0.25 \%$. Surgery is the main management in glaucoma cases in pediatric patients. Angle surgery such as goniotomy or trabeculotomy is the first choice in PCG, and could be performed in secondary childhood glaucoma, though it has a lower success rate. Filtration 
surgery and trabeculectomy are more recommended in most secondary glaucoma cases in children. A study conducted by Dolezal et al. [8] suggest that secondary childhood glaucoma in Peters' anomaly often requires multiple surgeries to control eye pressure. For the most part, a mere angle surgery does not show successful results due to the presence of iridocorneal adhesion. Installation of glaucoma drainage device (GDD) is sufficiently effective to control the eye pressure, though it requires a combination of vitrectomy and lensectomy to install the tube to posterior owing to anterior segment anomaly $[8,11,14]$. Our patient was only assigned for trabeculectomy procedure because of the presence of anterior synechia and shallow anterior chamber contributing to the difficulty in performing an angle surgery. Afterward, the patient was monitored periodically for IOP. A worse prognosis is expected when patients develop glaucoma and cataracts [2].

\section{Conclusions}

Timolol maleate therapy on the patient diagnosed with Peters' anomaly type I and secondary childhood glaucoma was not effective in reducing IOP. Surgical management, including trabeculotomy and trabeculectomy, could be performed to treat the glaucoma. Since the patient had anterior synechia and a shallow anterior chamber that cause angle surgery difficulties, only trabeculectomy could be performed. Cefadroxil syrup, paracetamol syrup, ophthalmic levofloxacin, ophthalmic prednisolone acetate, and ophthalmic homatropine could be prescribed for postoperative management. After 1-week post-surgery, blepharospasm, epiphora, photophobia, bleb on superior, subconjunctiva bleeding, buphthalmos, keratopathy, minimal corneal edema, anterior chamber with shallow image, and posterior synechia could still be found in the left eye anterior segment. Hence, the patient received ophthalmic timolol maleate, ophthalmic levofloxacin, ophthalmic prednisolone acetate tapering off, and ophthalmic homatropine. Further treatment strategy depends on the results obtained in the following control. Prognosis for the patient were quo ad vitam dubia ad malam, quo ad sanationam dubia ad malam, and quo ad functionam dubia ad malam.

\section{Declarations}

\section{Ethics approval}

Not applicable for case report.

\section{Patient's consent}

Informed written consent was taken from the parent of the patient to publish this case report and related evidence.

\section{Acknowledgments}

Thanks to the patient for giving consent to publish this case and the tertiary care center for referring the case.

\section{Conflict of interest}

Authors declare no conflict of interest.

\section{Funding}

This study received no funding.

\section{How to cite}

Imelda E and Gunawan F. Clinical management of a rare Peters' anomaly-induced secondary childhood glaucoma: A case report. Narra J 2021; 1(2):e53. ht tp://doi.org/10.52225/narraj.v1 i3.53. 


\section{References}

1. Weh E, Reis LM, Happ HC, et al. Whole exome sequence analysis of Peters anomaly. Human Genetics 2014; 133(12):1497-1511.

2. Ciralsky J, Colby K. Congenital corneal opacities: a review with a focus on genetics. Semin Ophthalmol 2007; 22(4):241-246.

3. Bhandari R, Ferri S, Whittaker B, et al. Peters anomaly: review of the literature. Cornea 2011; 30(8):939-944.

4. Kurilec JM, Zaidman GW. Incidence of Peters anomaly and congenital corneal opacities interfering with vision in the United States. Cornea 2014; 33(8):848-850.

5. Samara A, Eldaya RW. Ocular and brain imaging findings in Peters' anomaly: A case report and literature review. Radiol Case Rep 2020; 15(7):863-866

6. Shigeyasu C, Yamada M, Mizuno Y, et al. Clinical features of anterior segment dysgenesis associated with congenital corneal opacities. Cornea 2012; 31(3):293-298.

7. Yang LL, Lambert SR, Lynn MJ, et al. Surgical management of glaucoma in infants and children with Peters' anomaly: long-term structural and functional outcome. Ophthalmology 2004; 111(1):112-117.

8. Dolezal KA, Besirli CG, Mian SI, et al. Glaucoma and cornea surgery outcomes in peters anomaly. Am J Ophthalmol 2019; 208:367-375.

9. Hong J, Yang Y, Cursiefen C, et al. Optimising keratoplasty for Peters' anomaly in infants using spectral-domain optical coherence tomography. Br J Ophthalmol 2017; 101(6):820-827.

10. Spierer O, Cavuoto KM, Suwannaraj S, et al. Outcome of optical iridectomy in Peters anomaly. Graefes Arch Clin Exp Ophthalmol 2018; 256(9):1679-1683.

11. Heath DH, Shields MB. Glaucoma and Peters' anomaly. A clinicopathologic case report. Graefes Arch Clin Exp Ophthalmol 1991; 229(3):277-280.

12. Papadopoulos M, Vanner EA, Grajewski AL, et al. International Study of Childhood Glaucoma. Ophthalmol Glaucoma 2020; 3(2):145-157.

13. Thau A, Lloyd M, Freedman S, et al. New classification system for pediatric glaucoma: implications for clinical care and a research registry. Curr Opin Ophthalmol 2018; 29(5):385-394.

14. Mandal AK, Sulthana SS, Gothwal VK. Primary congenital glaucoma: trends in presentation over 3 decades at a tertiary eye care center in India. J Glaucoma 2020; 29(11):1095-1100.

15. Zieske JD. Corneal development associated with eyelid opening. Int J Dev Biol 2004; 48(8-9):903-911.

16. Chun AG. Peters' Anomaly. Available from: https://eyewiki.aao.org/Peters\%27_Anomaly. Accessed: 25 October 2021.

17. Salik I, Gupta A, Tara A, et al. Peters anomaly: A 5 -year experience. Paediatr Anaesth 2020; 30(5):577-583.

18. Harissi-Dagher M, Colby K. Anterior segment dysgenesis: Peters anomaly and sclerocornea. Int Ophthalmol Clin 2008; 48(2):35-42.

19. Seifi M, Walter MA. Axenfeld-Rieger syndrome. Clin Genet 2018; 93(6):1123-1130. 